\title{
MicroRNAs as Biomarkers for Personalized Antiplatelet Therapy
}

\author{
Mitchell J. Moon ${ }^{1,2}$ Karlheinz Peter ${ }^{1,2,3,4}$ \\ ${ }^{1}$ Atherothrombosis and Vascular Biology, Baker Heart and Diabetes \\ Institute, Melbourne, Victoria, Australia \\ ${ }^{2}$ Department of Cardiometabolic Health, University of Melbourne, \\ Melbourne, Victoria, Australia \\ ${ }^{3}$ Department of Medicine, Central Clinical School, Monash University, \\ Melbourne, Victoria, Australia \\ ${ }^{4}$ Department of Cardiology, The Alfred Hospital, Melbourne, Victoria, \\ Australia
}

Thromb Haemost 2021;121:1121.

MicroRNAs (miRNAs) are small, single-stranded, noncoding RNAs ( 22 nucleotides) with a central role in posttranscriptional regulation of gene expression. Notably, miRNAs also circulate in blood, predominantly in microparticles, acting to shuttle gene-regulating signals between cells. ${ }^{1,2}$ The presence of specific miRNAs in blood marks specific pathological changes in tissues and may therefore serve as a disease biomarker. $^{1,2}$ The search for specific miRNAs as disease biomarkers has exploded with over 27,000 total PubMed publications (4,724 from 2020 alone), demonstrating an enormous interest in circulating miRNAs in all areas of medicine.

Recent findings have shown the potential of miRNAs as biomarkers of platelet function and therapeutic efficacy. ${ }^{3}$ As the effectiveness of antiplatelet therapy varies substantially between patients, there is a strong need for biomarkers to aid in providing personalized antiplatelet therapy.

In this issue of Thrombosis and Haemostasis, Garcia et al ${ }^{4}$ identify an important role of miR-204-5p on in vitro platelet production and function. miR-204-5p-transfected megakaryocytes exhibited cytoskeletal alterations and generated more compact and more numerous platelet-like structures, which showed increased glycoprotein IIb/IIla expression and activation. The authors provide an intriguing insight into the genetic control of platelet production and function by miR204-5p. Importantly, the authors' data suggest miR-204-5p as a potential biomarker reporting on high platelet reactivity.

This in vitro work by Garcia et $\mathrm{al}^{4}$ supports the group's previous ex vivo work, ${ }^{5}$ which demonstrated the potential of miR-204-5p as a biomarker for high on-treatment platelet
Address for correspondence Karlheinz Peter, MD, PhD, Baker Heart and Diabetes Institute, 75 Commercial Road, Melbourne, 3004 Victoria, Australia (e-mail: karlheinz.peter@baker.edu.au).

reactivity, an indicator of future thrombotic events. As such, miR-204-5p promises to be a miRNA biomarker that reports on the effectiveness of antiplatelet therapy and will thus allow adjustments to dosage and treatment (changing to a more effective P2Y12 inhibitor). Thereby, this miRNA biomarker holds the promise to facilitate personalized antiplatelet therapy and exemplifies the potential use of circulating miRNAs in personalized medicine.

Conflict of Interest

None declared.

\section{References}

1 Diehl P, Fricke A, Sander L, et al. Microparticles: major transport vehicles for distinct microRNAs in circulation. Cardiovasc Res 2012;93(04):633-644

2 Garcia A, Dunoyer-Geindre S, Fish RJ, Neerman-Arbez M, Reny JL, Fontana P. Methods to investigate miRNA function: focus on platelet reactivity. Thromb Haemost 2021;121(04):409-421

3 Pedersen OB, Grove EL, Kristensen SD, Nissen PH, Hvas AM. MicroRNA as biomarkers for platelet function and maturity in patients with cardiovascular disease. Thromb Haemost 2021. Doi: 10.1055/s-0041-1730375

4 Garcia A, Dunoyer-Geindre S, Nolli S, Strassel C, Reny JL, Fontana P. miR-204-5p and platelet function regulation: insight into a mechanism mediated by CDC42 and GPIIbIIIa. Thromb Haemost 2021;120(09):1206-1219

5 Garcia A, Dunoyer-Geindre S, Nolli S, Reny J-L, Fontana P. An ex vivo and in silico study providing insights into the interplay of circulating miRNAs level, platelet reactivity and thrombin generation: looking beyond traditional pharmacogenetics. J Pers Med 2021;11(05):323 received

July 21,2021

accepted after revision

July 22, 2021

published online

July 26, 2021 (c) 2021. Thieme. All rights reserved. Georg Thieme Verlag KG,

Rüdigerstraße 14,

70469 Stuttgart, Germany
DOI https://doi.org/

10.1055/a-1559-0690. ISSN 0340-6245. 